Revue d'Études Françaises

$\mathrm{N}^{\mathrm{o}} 24(2020)$

DOI : $10.37587 /$ ref.2020.1.09

\title{
RADKA MUDROCHOVÁ
}

\section{La traduction des dénominations argotiques désignant l'argent à partir d'un corpus parallèle : l'exemple de la langue tchèque}

The objective of this article is to analyze the translations of the slang terms indicating money. The corpus of these terms is drawn from general language dictionaries such as Petit Robert, Larousse or Trésor de la langue française, as well as from specialized dictionary of slang (online): Dictionnaire de la Zone. Once the corpus has been developed: (1) its different terms are classified according to the lexicographic marking of the language register (such as : argotique, populaire, familier, etc.) ; (2) the translation of the chosen words (via the parallel corpus InterCorp) is compared and commented.

\section{Introduction}

L'objectif de cet article ${ }^{1}$ est d'analyser les traductions des dénominations argotiques/familières désignant «l'argent » en se focalisant sur la question des marques lexicographiques, des traductions et de leurs équivalents, employées par des corpus métalinguistiques choisis. L'article cherche notamment à savoir quels sont les équivalents substandard du mot «argent» en français et leurs traductions en tchèque, tout en prenant en compte les registres/niveaux de langue distincts.

Dans un premier temps de notre recherche, nous avons consulté le dictionnaire le Petit Robert (PR, en ligne, 2019) pour vérifier la définition et le concept de l'entrée «l'argent». Elle est introduite comme «toute sorte de monnaie (métallique, scripturale, papier-monnaie)» et complétée par l'affirmation «ce qui représente cette monnaie ». La définition se termine par l'énumération de synonymes, d'une part sans marque lexicographique : capital, fonds, fortune, monnaie, numérale, pécule, recette, ressource, richesse; d'autre part avec marque lexicographique fam. (familier) ou pop. (populaire) : blé, braise, cash, flouze, fric, galette, grisbi, jonc, maille, oseille, pépètes, pèze,

\footnotetext{
${ }^{1}$ Le présent article a bénéficié du soutien du projet SGS-2020-029.
} 
picaillon, pognon, rond, sou, thune, trèfle. Cette liste de base de dénominations désignant l'argent du registre familier/populaire provenant du dictionnaire le PR a été complétée par les données du Dictionnaire de la Zone dans l'objectif de constituer un corpus plus large, comptant au total 48 lexies.

Une fois le corpus élaboré : (1) ses différents termes sont classés selon le marquage lexicographique de registre de langue (comme : argotique, populaire, familier, etc.) proposé par les dictionnaires de langue générale ou spécialisés; (2) des mots choisis sont analysés via le corpus parallèle InterCorp ${ }^{2}$ (créé et géré par l'Institut du Corpus national tchèque (disponible sur : korpus.cz) de l'Université Charles de Prague) permettant de comparer des traductions de textes dans plusieurs langues incluant et offrant la possibilité de distinguer les différentes ressources de textes (belles lettres, domaine juridique, sous-titres de films, etc.). Néanmoins, la présente contribution ne se concentre que sur la traduction franco-tchèque sans prendre en compte les domaines distingués dans le corpus.

\section{Marquages lexicographiques et registre de langue / niveaux de langue}

Avant de présenter les analyses effectuées, nous trouvons nécessaire d'aborder la question de registres/niveaux de langue qui se rapporte aux marquages lexicographiques inclus dans les entrées des dictionnaires. Pour rappel, le Trésor de la langue francaise informatisé définit d'un côté les registres de langue: «usages divers qui sont faits de cette langue (de ce discours) selon les milieux où elle est employée ou selon les situations psychosociologiques dans lesquelles se trouve l'émetteur » et de l'autre côté, les niveaux de langue: "chacune des variétés stylistiques d'une langue, distinguées plus ou moins arbitrairement, à l'aide de marques temporelles (p. ex. vx), spatiales (p. ex. région.) et socio-culturelles (p. ex. pop.) ». Dans ce contexte, les linguistes Nicole et Jean Tournier (2009: 306) soulignent que «malgré des confusions encore courantes entre les deux termes», certains linguistes les distinguent systématiquement (p.ex.: Grevisse, 1986: 18, Podhorná-Polická, 2007 : 48, Tournier, 2009 : 30). Selon Tournier (ibid), le terme registre se rapporte au discours, tandis que le niveau au lexique. En outre, A. Podhorná-Polická (2007 : 48) illustre cette distinction par deux axes de conditionnements socioculturel et situationnel (cf. figure 1). Néanmoins, elle

\footnotetext{
${ }^{2}$ Pour apprendre plus sur le corpus InterCorp, cf. Nádvorníková (2017a).
} 
admet que les marques de la provenance sociale et les marques stylistiques/situationnelles posent problème à cause de leur subjectivité d'attribution (Podhorná-Polická, 2009 : 98).

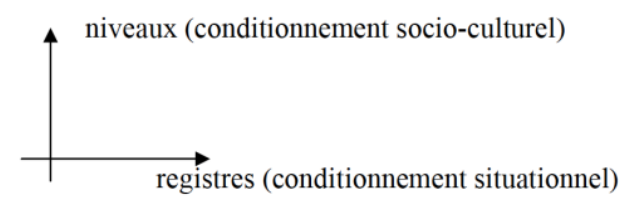

Figure 1 : Niveaux vs registres de langue selon Podhorná-Polická (2007)

La subjectivité d'attribution de marques lexicographiques, qu'elles soient situationnelles ou socioculturelles, pose des problèmes également aux lexicographes, aux rédacteurs de dictionnaires, car l'application des marques est très diverse (cf. par exemple Gadet, 2003), ce qui peut être démontré par l'introduction du tableau 1 (cf. infra).

En effet, nous avons vérifié les 48 lexies dans les dictionnaires de langue générale (Petit Robert, Larousse, Wiktionnaire) tout en incluant les données du Dictionnaire de la Zone que nous tenons pour argotiques, même si les marques lexicographiques n'y sont pas explicitement indiquées. En outre, la lettre « $\mathrm{N}$ » désigne l'absence de la lexie dans le dictionnaire en question et la lettre « $\mathrm{O}$ » sa présence sans qu'il y ait de précision concernant le registre/niveau de langue. En revanche, les précisions indiquées par les dictionnaires sont explicitement mentionnées dans le tableau, souvent sous forme abrégée ${ }^{3}$.

Pour chaque mot, nous avons décidé de relever une marque lexicographique dominante. Cela veut dire que s'il y a une marque employée par tel ou tel dictionnaire au moins deux fois, nous l'avons considérée comme dominante. Le bleu indique la marque «familier», le vert clair désigne « argotique », le vert foncé représente «argotique» avec l'exclusivité de la présence dans le Dictionnaire de la Zone, l'orange est assigné à la marque "populaire » et le gris signale les cas «sans décision», car leur marquage est trop dispersé. Finalement, les marquages en rouge ne permettent que de souligner la diversité du traitement lexicographique.

\footnotetext{
${ }^{3}$ Voici le répertoire des abréviations utilisées dans le tableau $:$ arg. $=$ argotique, pop. $=$ populaire, fam. = familier, CA = Canada (emploi limité au Canada), Q. = Québec (emploi limité au Québec), $\mathrm{SM}=$ sans marque lexicographique, $\mathrm{vx}=$ vieux, $\bmod .=$ moderne, anglic. $=$ anglicisme .
} 


\begin{tabular}{|c|c|c|c|c|c|c|c|c|c|}
\hline Lexie & Zone & $P R$ & Wiktionnaire & Larousse & Lexie & Zone & $P R$ & Wiktionna & Larousse \\
\hline artiche & 0 & $\mathrm{~N}$ & arg. & \begin{tabular}{|l|l}
$\mathrm{N}$ \\
\end{tabular} & mornifle & 0 & $\mathrm{~N}$ & pop. vieilli & pop. et vieux \\
\hline bidou & $\mathrm{N}$ & $\mathrm{N}$ & fam. Q. & $\mathrm{N}$ & motton & $\mathrm{N}$ & $\mathrm{CA}$ & fam.Q. & Q/SM \\
\hline biff & 0 & $\mathrm{~N}$ & $\mathrm{~N}$ & $\mathrm{~N}$ & naimo/némo & 0 & $\mathrm{~N}$ & $\mathrm{~N}$ & $\mathrm{~N}$ \\
\hline bifton/biffeton & 0 & fam. & arg. & $\mathrm{N}$ & neuthu/neutu & 0 & $\mathrm{~N}$ & $\mathrm{~N}$ & $\mathrm{~N}$ \\
\hline billes & 0 & $\mathrm{~N}$ & $\mathrm{~N}$ & $\mathrm{~N}$ & oseille & 0 & fam. & fam. & pop. \\
\hline blé & 0 & fam. & arg. & pop. & osier & $\mathrm{N}$ & $\mathrm{N}$ & arg. vieilli & $\mathrm{N}$ \\
\hline boule & 0 & fam. & arg. & $\mathrm{N}$ & patate & 0 & fam. & pop. & $\mathrm{N}$ \\
\hline braise & $\mathrm{N}$ & arg. vieilli & arg. & arg. & pélaud & $\mathrm{N}$ & $\mathrm{N}$ & arg. & $\mathrm{N}$ \\
\hline caillasse & 0 & $\mathrm{~N}$ & $\mathrm{~N}$ & $\mathrm{~N}$ & pépète/pépette & 0 & fam. et vieilli & fam. & $\mathrm{N}$ \\
\hline cash & $\mathrm{N}$ & anglic. fam & fam. & fam. & pèze/pèse & 0 & arg. & arg. & arg. \\
\hline cenne & $\mathrm{N}$ & \begin{tabular}{|l|}
$\mathrm{N}$ \\
\end{tabular} & fam. Q. & $\mathrm{N}$ & piastre & $\mathrm{N}$ & $0 / \mathrm{SM}$ & fam. $Q$. & fam. CA \\
\hline flouze & 0 & pop. & arg. & pop. & picaillon & $\mathrm{N}$ & fam. vieilli & fam. & $\mathrm{N}$ \\
\hline foin & $\mathrm{N}$ & $\mathrm{N}$ & fam. Q. & $\mathrm{N}$ & plaque & 0 & $\mathrm{~N}$ & arg. & $\mathrm{N}$ \\
\hline fraîche & 0 & $\mathrm{~N}$ & arg. & pop. & pognon & 0 & fam. & fam. & pop. \\
\hline fric & 0 & fam. & fam. & pop. & quibus & $\mathrm{N}$ & $\mathrm{N}$ & arg. & $\mathrm{N}$ \\
\hline galette & 0 & fam. vieilli & pop. & fam. vieilli & radis & $\mathrm{N}$ & fam. & pop. & $\mathrm{N}$ \\
\hline gengen & 0 & \begin{tabular}{|l|l}
$\mathrm{N}$ & \\
\end{tabular} & $\mathrm{N}$ & \begin{tabular}{|l|l}
$\mathrm{N}$ \\
\end{tabular} & rond & 0 & fam. & arg. & $\mathrm{N}$ \\
\hline genhar & 0 & $\mathrm{~N}$ & verlan & $\mathrm{N}$ & sou & $\mathrm{N}$ & fam. & cour. & fam. \\
\hline grisbi & $\mathrm{N}$ & arg. & arg. & arg. & talbin & 0 & $\mathrm{~N}$ & arg. & $\mathrm{N}$ \\
\hline jonc & $\mathrm{N}$ & vieilli & $\mathrm{N}$ & pop. & thune/tune & 0 & arg. vx + mod. & arg. & arg. XIXe/fam. (Suisse) \\
\hline keusse & 0 & $\mathrm{~N}$ & arg. & $\mathrm{N}$ & tomates & $\mathrm{N}$ & $\mathrm{N}$ & fam. & $\mathrm{N}$ \\
\hline khalis & 0 & $\mathrm{~N}$ & $\mathrm{~N}$ & $\mathrm{~N}$ & trèfle & $\mathrm{N}$ & fam. et vieux & arg. & arg. \\
\hline love/lové & 0 & $\mathrm{~N}$ & arg. & $\mathrm{N}$ & yeuma & 0 & $\mathrm{~N}$ & $\mathrm{~N}$ & $\mathrm{~N}$ \\
\hline maille & 0 & fam. & arg. & $0 / \mathrm{SM}$ & zeillo/zeyo & 0 & $\mathrm{~N}$ & $\mathrm{~N}$ & $\mathrm{~N}$ \\
\hline
\end{tabular}

Tableau 1 : Marquages lexicographiques des dénominations désignant l'argent dans les dictionnaires choisis

Les résultats récapitulatifs de cette distinction de marquages lexicographiques prédominants sont illustrés par le graphique 1 .

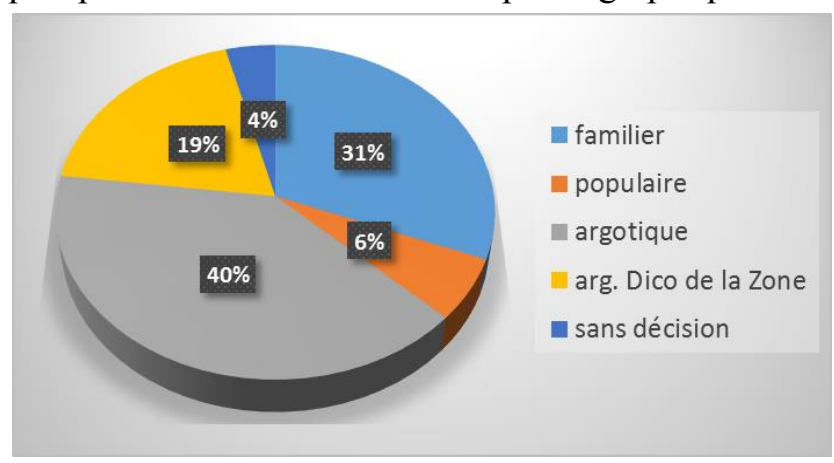

\section{Graphique 1 : Répartition du marquage lexicographique}

Le graphique 1 révèle que c'est le marquage «argotique » qui prédomine dans notre corpus de recherche, suivi de «familier». 


\section{Traduction des mots français substandard désignant l'argent en tchèque}

Pour choisir le corpus final de notre recherche de traduction, nous avons sélectionné les mots qui sont présents dans au moins trois des dictionnaires cités supra, qui respectent en même temps le marquage lexicographique substandard (fam., arg., pop.) et sont les plus fréquents dans le dictionnaire $B o b^{4}$. Ainsi, nous avons restreint notre corpus au nombre de 15 items dont les équivalents tchèques ont été recherchés dans le corpus Treq, une application faisant partie du Corpus national tchèque de l'Université Charles de Prague, disponible sur: korpus.cz. Treq est une collection de dictionnaires bilingues tchèque / anglais-langue étrangère, construite automatiquement à partir du corpus parallèle InterCorp permettant la traduction en 39 langues ${ }^{5}$. La langue cible/source est soit le tchèque soit l'anglais et on peut les combiner avec toutes les autres langues. Les types de textes inclus sont très variés : belles lettres, textes juridiques, sous-titres de films et il est possible de limiter la recherche au corpus de textes choisis. Néanmoins, nous n'avons pas utilisé cette fonctionnalité, car notre objectif ne consistait pas dans la distinction des traductions par domaine, mais nous voulions obtenir une vue globale de la traduction des termes substandard et comparer notamment les termes français et leurs homologues tchèques pour ce qui est des marques diastratiques proposées par des corpus métalinguistiques. De ce point de vue, nous sommes restée plutôt dans des analyses quantitatives en optant pour la présentation des exemples et des cas particuliers qui seront présentés infra.

Pour respecter les objectifs et la méthodologie fixés, nous avons créé, dans un premier temps, un tableau de traduction et nous avons noté chaque présence de l'équivalent tchèque par le chiffre 1 . Ainsi, nous avons pu établir les traductions possibles de chaque mot, sans compter le nombre d'occurrences de ces traductions pour chaque lexème. Le tableau 2 résume les résultats obtenus et contient également un graphique avec les résultats globaux indiquant le nombre d'emploi de l'équivalent, en pourcentage, dans l'ensemble des traductions. La traduction la plus fréquente est celle de «prachy» $(87 \%)$,

\footnotetext{
${ }^{4} \mathrm{http}: / / \mathrm{www}$.languefrancaise.net/Bob/Introduction

${ }^{5}$ À savoir : ar, be, bg, ca, da, de, el, en, es, et, fi, fr, he, hi, hr, hu, is, it, ja, lt, lv, mk, ms, mt, nl, no, pl, pt, ro, ru, sk, sl, sq, sr, sv, tr, uk, vi, cs (pour voir la taille de ces différents sous-corpus, veuillez consulter http://ucnk.ff.cuni.cz/intercorp/?req=page:info, cité par Nádvorníková (2017b : 23).
} 
relevée dans 13 cas sur 15 de notre corpus de lexies. En effet, seuls les mots pèze et patate ne possèdent pas l'équivalent tchèque "prachy ». La traduction la plus standard, celle de « peníze » (argent), compte $67 \%$, étant proposée dans les traductions des mots suivants : bifton, blé, cash, fric, galette, oseille, pèze, pognon, rond, thune. Nous notons les pourcentages moins importants (entre $33 \%$ et $20 \%$ ) pour les traductions babka, škvára, balik, hotovost, prašule. Les autres cas répertoriés dans le tableau restent marginaux, avec une ou deux traductions relevées.

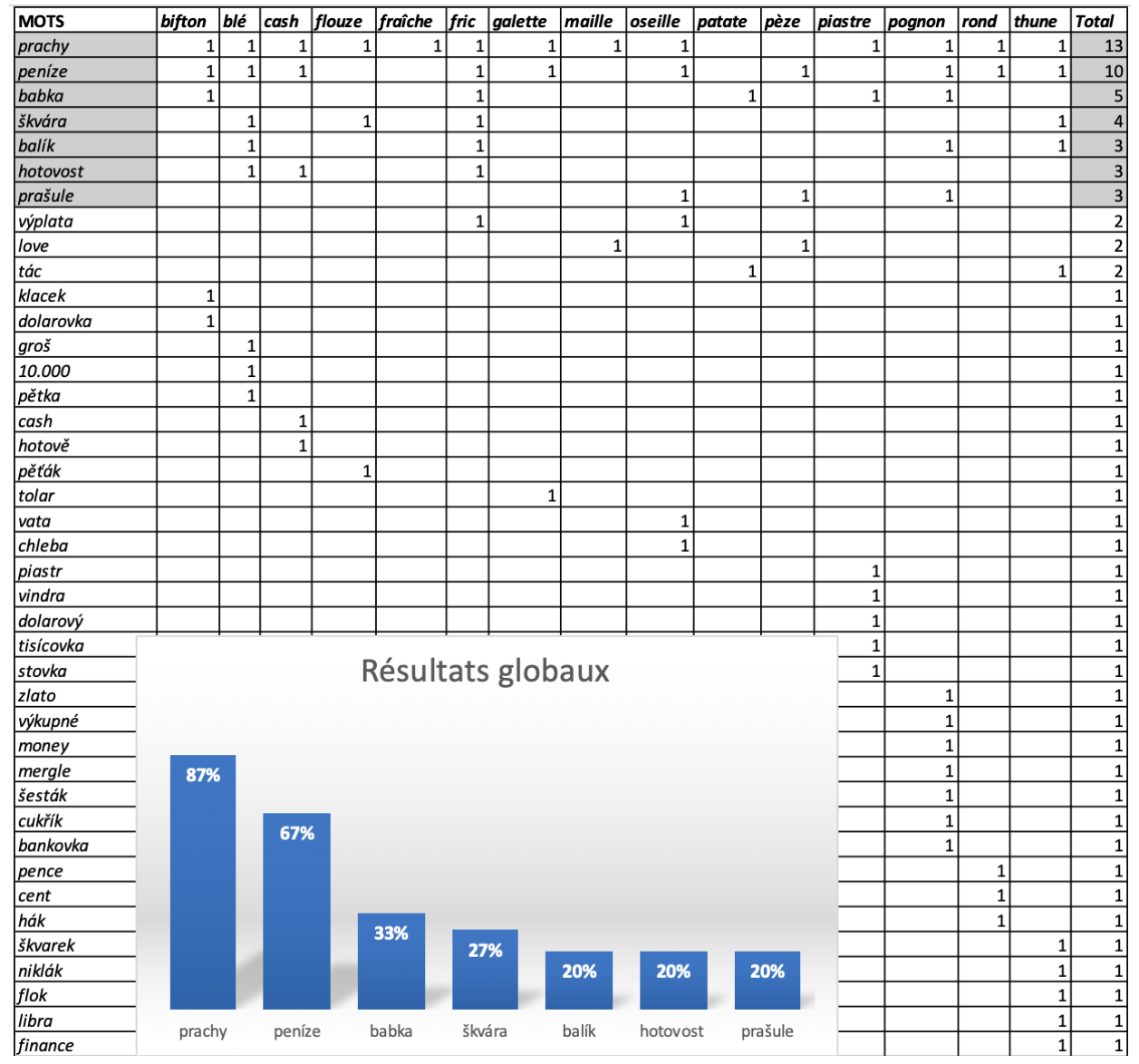

Tableau 2 : Résultats de traduction dans le corpus Treq 
En prenant en compte les résultats globaux du graphique supra, nous avons effectué, dans un deuxième temps, une recherche de vérification quant aux traductions tchèques dans le dictionnaire monolingue Le dictionnaire $d u$ tchèque contemporain (Lingea), disponible gratuitement en ligne, qui comporte des marquages lexicographiques éventuels. Pour le mot «balík» nous trouvons le marquage «familier», le mot « love» comporte la marque « argotique», et dans deux cas («prachy », «babka») nous notons l'abréviation « expr. » qui désigne en milieu linguistique tchèque «lexique marqué » (cf. PodhornáPolická, 2009 : 97). Un mot est complètement absent du dictionnaire et les cinq autres lexies restent sans marquages lexicographiques. Cette décision semble pour les unes («hotovost », «peníze », « výplata ») justifiée, cependant pour les autres (« škvára », « tác ») surprenante. En effet, les mots « škvára ( au sens propre : fraisil) qui correspond au fric, et « tác » (au sens propre : plateau) qui se traduit comme brique, sont considérés par le Wikislovník comme des mots d'origine argotique. Toutefois, ce marquage est absent dans le dictionnaire de référence du tchèque contemporain Lingea.

Dans un troisième temps, nous nous permettons de signaler plusieurs cas particuliers. La première particularité, ou plutôt curiosité, que nous avons rencontrée, est celle de la traduction du mot "tác » (signifiant plateau en français) : en effet, dans le corpus Treq, il est majoritairement traduit par la lexie brique. Néanmoins, le chiffre qui accompagne à chaque fois cette lexie n'est pas toujours traduit de manière équivalente, car « tác » correspond à mille, tandis que brique exprime, en argot, d'après le Wiktionnaire: "un million d'ancien français (dix mille nouveaux francs français, soit 1524 euros) ».

L'hésitation dans la traduction de cette notion peut être illustrée par l'extrait du corpus InterCorp (cf. figure 2 infra) représentant la traduction «tác»brique. Un tableau avec le comptage correspondant en tchèque et en français (en nouveaux francs français) a été inséré au milieu de cet extrait. Cela veut dire que pour la langue tchèque, nous avons remplacé « tác » par 1.000 et son homologue français a été compté en nouveaux francs, donc en 10.000. 


\begin{tabular}{|c|c|c|c|c|}
\hline InterCorp v9-Czecho & & & & InterCorp v9 - Frenche \\
\hline Tak pèt tácû jistê, co ? & 5.000 & 10.000 & Fuel-Audience & Une brique, au moins, non ? \\
\hline "To prijde lehce na takových padesát tácí. " & 50.000 & 50.000 & Aprisot:Dama & - Ça doit bien aller chercher dans les cinq briques. \\
\hline Tento tejden jsme yydèlali 20 tácú. & 20.000 & 200.000 & fürtites & On a fait plus de 20 briques la 1ère semaine. \\
\hline Vrazil jsem do toho 20 tácu. & 20.000 & 200.000 & 7uвтітеs & $J^{\prime}$ ai claqué vingt briques dessus. \\
\hline Čekal jsem na to tejden, tak by za tèch padesát tácû méla stát. & 50.000 & 500.000 & fuemress & Pour 50 briques, elle a intérêt à être bonne. \\
\hline A tohle všechno na celý vikend ....... za pouhý tri tácy. & & & fuBtitus & Pour tout, nous parlons de trois briques pour un week-end ... \\
\hline - Asi 37 tácú. & 37.000 & 370.000 & fütrites & -37 briques. \\
\hline Osmdesát tácû. & 80.000 & 800.000 & fuertines & 80 briques ! \\
\hline - Tu se 40 ticy. & 40.000 & 200.000 & Juertines & - Une avec 20 briques ? \\
\hline -Záádných 40 tácú neexistuje. & 40.000 & 200.000 & fütrus & -IIn'y a pas de 20 briques. \\
\hline 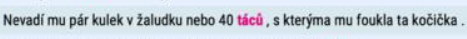 & 40.000 & 200.000 & fustrites & II pardonne les pruneaux et les 20 briques empochées. \\
\hline Kde je ten zasranej Dorfman , ten häzl, co mẻ stoji 30 tácú ! & 30.000 & 300.000 & funtrites & Ce salaud m' a déàa coưté 30 briques! \\
\hline
\end{tabular}

Figure 2 : Traduction « tác » - brique dans le corpus InterCorp

Nous pouvons observer que l'équivalence des chiffres exprimés en tchèque et en français n'est respectée que dans un seul cas (mis en gras). Quant aux autres cas, les chiffres sont toujours plus élevés dans les textes français.

Deux autres lexies françaises, à savoir fric et pognon, méritent aussi une attention particulière. Le premier représente le mot qui désigne l'argent avec la plus grande fréquence dans le corpus Treq (1639 occ.). Même s'il est marqué au niveau lexicographique en français, sa traduction tchèque renvoie à $41 \%$ au registre standard avec l'équivalent « peníze » (argent). Cependant, la traduction tchèque la plus fréquente, avec $44 \%$, reste également le mot marqué, « prachy ». L'autre mot, pognon, est cité pour son grand nombre de variétés de traduction en tchèque, qui se trouvent répertoriées dans le tableau infra (cf. tableau 3). Toutefois, de nombreuses possibilités de traduction restent marginales dans son emploi car son nombre d'occurrences dans le corpus est très faible.

\begin{tabular}{|l|r|}
\hline \multicolumn{2}{|c|}{ pognon (172) } \\
\hline prachy & $49 \%$ \\
\hline peníze & $33 \%$ \\
\hline balík & $1 \%$ \\
\hline babka & $1 \%$ \\
\hline zlato & $1 \%$ \\
\hline výkupné & $0,60 \%$ \\
\hline prašule & $0,60 \%$ \\
\hline money & $0,60 \%$ \\
\hline mergle & $0,60 \%$ \\
\hline šesták & $0,60 \%$ \\
\hline cukŕík & $0,60 \%$ \\
\hline bankovka & $0,60 \%$ \\
\hline
\end{tabular}

Tableau 3 : Variétés de traduction du mot pognon 
Il est à observer que la lexie pognon est traduite principalement par « prachy » (mot marqué) avec $49 \%$, suivi de « peníze » (non marqué), donc par les deux équivalents les plus fréquents dans l'ensemble du corpus. C'est la raison pour laquelle nous avons ensuite effectué une recherche inverse en tapant les deux lexies tchèques, "prachy » et "peníze », dans le corpus Treq pour avoir l'échantillon de leurs traductions françaises. Les deux graphiques suivants (cf. les graphiques 2 et 3 ) résument le pourcentage des équivalents français relevés dans le cas de chaque lexème.

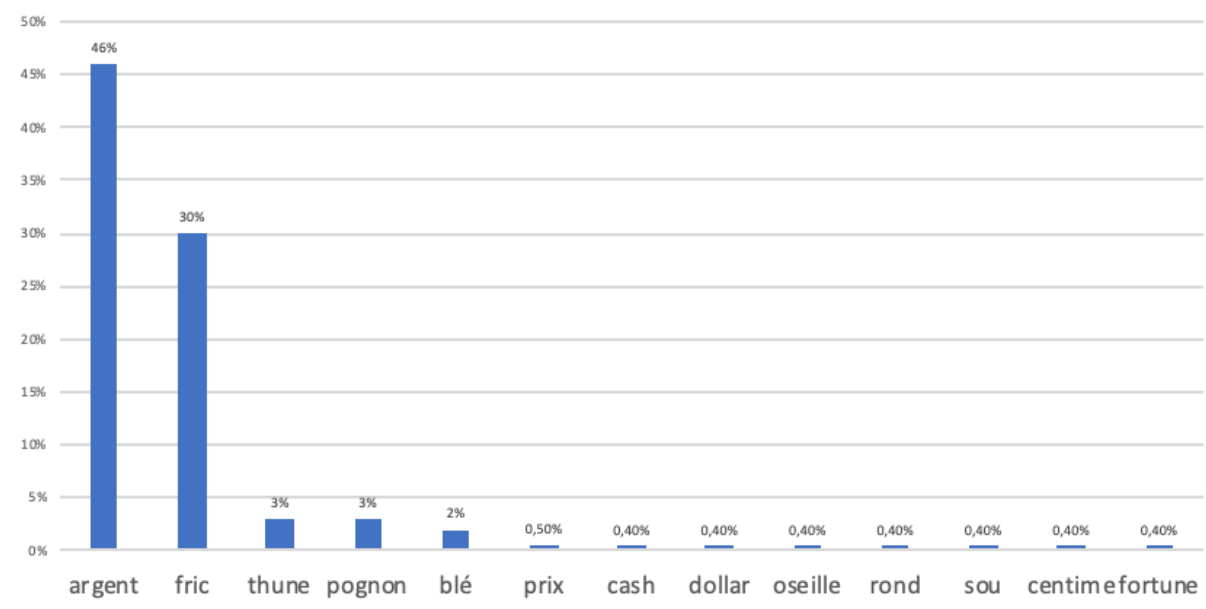

Graphique 2 : Traduction de «prachy » en français dans le corpus Treq

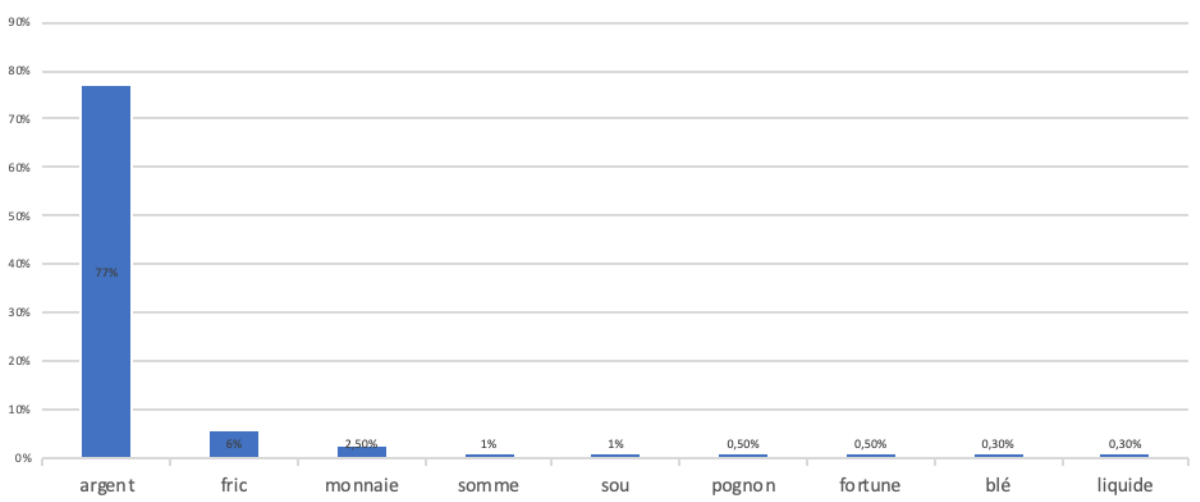

Graphique 3 : Traduction de «peníze » en français dans le corpus Treq 
Force est de constater que la traduction la plus fréquente des deux lexies est le groupe nominal «l'argent», un équivalent du registre standard, alors que la lexie « prachy » appartient en tchèque au niveau familier, comme le soulignent, par exemple, le dictionnaire Wikislovnik et Le Dictionnaire de la langue tchèque contemporaine. En même temps, le nombre d'occurrences des autres traductions reste marginal.

\section{Conclusion}

Les études effectuées permettent de tirer plusieurs conclusions concernant la traduction des dénominations françaises désignant l'argent en tchèque. Premièrement, nous constatons que les marques lexicographiques appliquées par les dictionnaires analysés ne sont pas toujours identiques. Néanmoins, toutes les lexies de notre corpus font partie du français non standard, voire substandard. Or, les linguistes tchèques optent plus souvent pour la marque « expr. » = « lexique marqué », probablement pour éviter toute hésitation entre les marques : familier, argotique et populaire.

Deuxièmement, les lexies françaises de notre corpus sont le plus souvent traduites en tchèque par le mot "prachy» (dérivé en tchèque de "prach» signifiant poussière). Cependant, la traduction «de retour» a montré que ce dernier était le plus fréquemment traduit en français par un lexème neutre, standard «peníze » (argent). Cet équivalent représente également la deuxième traduction la plus présente dans le corpus, même si le nombre de ses occurrences n'a pas été pris en compte dans l'analyse des résultats globaux.

Troisièmement, nous avons pu suivre plusieurs cas particuliers, notamment le lexème fric, qui a le plus d'occurrences dans le corpus et dont la traduction en tchèque oscille entre les deux équivalents cités supra, à savoir «prachy » et «peníze ».

Finalement, cette étude a non seulement permis de révéler des possibilités de traduction des dénominations de l'argent «marquées», mais aussi, et surtout, de percevoir une frontière très fine entre le registre familier et standard. Si le mot "prachy », marqué en tchèque, est majoritairement lié dans le corpus au fric, le lexème non marqué " peníze » possède deux traductions avec des nombres d'occurrences concurrentiels : «fric » $(46 \%)$ et «argent» $(30 \%)$. Évidemment, il ne s'agit que d'une étude préliminaire, plutôt quantitative, qui pourrait être encore développée de différentes manières. 


\section{Bibliographie}

GADET Françoise (2003), La variation sociale en français, Paris, Ophrys. GREVISSE Maurice, GOOSE André (1986), Le Bon usage, Paris, Duculot. NÁDVORNÍKOVÁ Olga (2017a), «Le corpus multilingue InterCorp : nouveaux paradigmes de recherche en linguistique contrastive et en traductologie », Studii de lingvistică, $\mathrm{n}^{0}$ 7, p. 67-88.

NÁDVORNÍKOVÁ Olga (2017b) «Pièges méthodologiques des corpus parallèles et comment les éviter ", Corela [en ligne], HS-21 | 2017, mis en ligne le 20 février 2017, URL : http://corela.revues.org/4810 (consulté 21.02.2020.). DOI: $10.4000 /$ corela.4810

PODHORNÁ-POLICKÁ Alena (2007), Peut-on parler d'un argot des jeunes? Analyse lexicale des universaux argotiques du parler de jeunes en lycées professionnels en France (Paris, Yzeure) et en République tchèque (Brno), Thèse en cotutelle, Paris-Brno.

PODHORNÁ-POLICKÁ Alena (2009), Universaux argotiques des jeunes. Analyse linguistique dans les lycées professionnels français et tchèques, Brno, Masarykova univerzita.

TOURNIER Nicole, TOURNIER Jean (2009), Dictionnaire de lexicologie française, Paris, Ellipses.

\section{Sitographie}

Trésor de la langue française, http://atilf.atilf.fr (consulté 23.06.2019.).

Larousse, https://larousse.fr (consulté 02.07.2019.).

Wikislovník, https://cs.wiktionary.org/wiki/Wikislovn\%C3\%ADk:Hlavn\%C3\%AD_stran a (consulté 23.11.2019.).

Wiktionnaire, https://fr.wiktionary.org/wiki/Wiktionnaire:Page_d'accueil (consulté 23.11.2019.).

Le Petit Robert, https://lerobert.com (consulté 21.05.2019.).

Dictionnaire de la Zone, https://www.dictionnairedelazone.fr (consulté 06.01.2020.).

Slovník současné češtiny, https://www.nechybujte.cz/slovnik-soucasne-cestiny (consulté 23.11.2019.).

Dictionnaire de 1'argot Bob, https://www.languefrancaise.net/Bob/Introduction (consulté 20.03.2019.). 
InterCorp, https://kontext.korpus.cz/corpora/corplist?requestable=1 (consulté 05.01.2020.).

Treq, https://treq.korpus.cz (consulté 05.01.2020.).

RADKA MUDROCHOVÁ

Université Charles de Prague, Université de Bohême de l'Ouest

Courriel : rfridrichova@seznam.cz 